\title{
REVISIÓN DE INVESTIGACIONES SOBRE ENFERMEDADES MUSCULOESQUELÉTICAS RELACIONADAS CON EL TRABAJO DE 2017- 2020
}

\author{
RESEARCH REVIEW OF WORK RELATED MUSCULOSKELETAL DISEASES 2017-2020
}

\author{
Luis Fernando Mesta Carrillo* \\ Beatriz Sibaja Terán ${ }^{* *}$
}

\begin{abstract}
Resumen: La manifestación de las molestias, dolores o enfermedades musculoesqueléticas generadas por el sobreesfuerzo o desgaste físico de los tejidos del aparato locomotor es una de las principales preocupaciones del especialista en ergonomía, por lo tanto, es necesario contar con información actualizada sobre los hallazgos recientes con carácter científico para dar soluciones en los centros de trabajo. El objetivo fue realizar una revisión bibliográfica en PubMed de los últimos tres años para conocer las características específicas de los países que estudian estos aspectos y qué han encontrado al respecto. La revisión contempla el periodo de marzo 2017 a marzo 2020 sobre las enfermedades musculoesqueléticas en países de los cinco continentes y que dan información de la exposición que se genera por riesgos no ergonómicos, lo que puede ser trascendente para los especialistas en esta área para evitar o corregir las fuentes generadoras desde un enfoque basado en la evidencia.
\end{abstract}

Palabras clave: Ergonomía, enfermedades musculoesqueléticas, salud laboral, enfermedades profesionales.

\begin{abstract}
The presence of musculoskeletal discomfort, pain or disease generated by overstrain or physical wear of the tissues of the locomotor system is one of the main concerns of the Ergonomics specialists, therefore, it is necessary to have updated information on recent findings with scientific character, to give solutions according to the urgencies that appear in the work centers. Covering this objective, a bibliographic review of the last three years was carried out in PubMed to find out the characteristics of countries where the studies occurred, workers who are they focused on, and the anatomical sites related to the studied disease. This work provides a bibliographic concentration of studies published from March 2017 to March 2020 about musculoskeletal diseases in the context of research focused on ergonomics. Information from five continents was included, as well as information about the exposure generated by non-ergonomic risks. This information could be important for specialists who are interested in knowing the latest sources from an evidence-based approach.
\end{abstract}

Keywords: Ergonomics, musculoskeletal diseases, occupational health, occupational diseases.

\footnotetext{
*Escuela Nacional de Medicina y Homeopatía, Instituto Politécnico Nacional. Ciudad de México, México. Correo electrónico: mclfernandomc@gmail.com. Orcid: https://orcid.org/oooo-00o2-43967321

**Escuela Nacional de Medicina y Homeopatía, Instituto Politécnico Nacional. Ciudad de México, México. Correo electrónico: bsibajat@ipn.mx. Orcid: https://orcid.org/oooo-0002-0514-6119. Autor de correspondencia.
} 


\section{Introducción}

En ergonomía es importante utilizar datos con rigor científico para que los especialistas tomen decisiones asertivas acerca de las medidas que puedan solucionar problemas y prevenir enfermedades musculoesqueléticas en la población trabajadora. La ergonomía es un campo multidisciplinar y su objetivo incluye la prevención de riesgos laborales. Esto construye una relación con la investigación en ciencias de la vida que permite comprender los cambios fisiológicos, biomecánicos y psicofísicos en el trabajador cuando está expuesto a actividades disergonómicas (Llaneza, 2009). Se reconoce que los métodos en ergonomía son elementales para mostrar una problemática de un lugar de trabajo, sin embargo, los cambios que se generen pueden ser neutrales o tener poco impacto si no se considera el contexto laboral; de ahí que sea importante registrar y evaluar los cambios según la perspectiva de la salud (Westgaard \& Winkel, 1997).

\section{Objetivo general}

Revisar las últimas investigaciones en ergonomía y trastornos musculoesqueléticos en el trabajo publicadas en la base de datos PubMed de la Biblioteca Nacional de Medicina de los Institutos Nacionales de Salud de EE.UU.

\section{Objetivos específicos}

- Revisar publicaciones en una base de datos científica sobre ergonomía, dolor y/o trastornos del sistema musculoesquelético generados por el trabajo.

- Seleccionar bibliografía con rigor científico sobre la presencia de enfermedades musculoesqueléticas que afecten a los trabajadores en el desempeño de sus tareas.

- Describir el dolor o la enfermedad musculoesquelética y el tipo de población activa afectada.

\section{Materiales y métodos}

Es una revisión de la literatura científica que incluye publicaciones de cualquier país, todas ellas centradas en el dolor relacionado con el trabajo o los trastornos musculoesqueléticos relacionados con el trabajo. Se utilizó el diseño de revisión de afrontamiento de artículos publicados de marzo de 2017 hasta marzo de 2020 exclusivamente en el motor de búsqueda Medline PubMed, en el código abierto proporcionado por la Biblioteca Nacional de Medicina de los Estados Unidos.

Criterios de selección

Criterios de inclusión: El artículo debe estar basado en evidencia científica, describe el año de 
publicación, debe ser publicado en inglés, debe especificar el país donde se realizó la investigación, así como indicar una metodología rigurosa para recolectar y analizar los resultados. Se debe incluir el tipo de población activa, así como información sobre el sitio anatómico estudiado, e información específica sobre el dolor estudiado y/o enfermedades del sistema musculoesquelético estudiadas.

Criterios de exclusión: Se excluyeron las publicaciones con datos faltantes sobre el tipo de población, país o faltantes en la descripción metodológica o la ausencia del instrumento para explorar el malestar musculoesquelético. No se consideraron tesis, actas de congresos, manuales o guías de referencia.

Criterios de eliminación: Se eliminaron de la segunda parte del análisis los artículos que no incluían en su metodología ningún instrumento validado para evaluar el dolor o medir la intensidad de los factores ergonómicos que pudieran estar relacionados con el dolor o malestar estudiado.

\section{Procedimiento}

Las palabras clave aplicadas fueron: "enfermedades musculoesqueléticas relacionadas con el trabajo", "dolor" y "ergonomía". Todos ellos son descriptores de las ciencias de la salud del vocabulario del tesauro de Medical Subject Headings (MeSH). Según los criterios de selección, la información fue registrada en el programa Excel para realizar el análisis descriptivo en donde se clasificaron según el tipo de artículo, la población de estudio, el país donde se realizó el estudio y la principal contribución al campo de la ergonomía.

Posteriormente, fueron etiquetados y clasificados en cuatro grupos generales: artículos de investigación originales, informes breves/de casos, revisión sistemática y metanálisis, y revisiones narrativas/bibliográficas y otros. Asimismo, según el tipo de población ocupada se distribuyeron en cuatro grupos: médicos cirujanos, prestadores de servicios de salud, administrativos, manufactura, construcción y otros. Una vez seleccionados los artículos, se examinó el texto completo para identificar los instrumentos utilizados en la evaluación del dolor o enfermedad musculoesquelética; También, se registraron los datos generales estudiados sobre el dolor o la enfermedad, incluido el sitio anatómico.

\section{Resultados y discusión}

Según los criterios de búsqueda, el sistema mostró 82 artículos; cuatro de ellos fueron excluidos por falta de criterio. Todos los resúmenes y citas de esos artículos se recuperaron en una hoja de cálculo de Excel para incluirlos en la revisión. De los 78 artículos incluidos, 63 (80,76\%) fueron investigaciones originales, incluidos ensayos clínicos, estudios de prevalencia y otros estudios intervencionistas. Por otro lado, 4 artículos $(5,12 \%)$ fueron casos/informes breves, 7 (8,97\%) fueron revisiones sistemáticas y metanálisis y $4(5,12 \%)$ tipo narrativa. En cuanto a la población de estudio, el 20,51\% (16) eran médicos de una amplia variedad de especialidades quirúrgicas; el 23,07\% (18) se realizaron en otros 
proveedores de atención de la salud, como odontólogos, enfermeras y técnicos en imagen, el $14,10 \%$ (11) se realizaron en trabajadores de actividades mayoritariamente administrativas o informáticas; y el 42,30\% (33) se realizaron sobre trabajadores de actividades predominantemente físicas, como manufactura, construcción o agricultura. Todos los artículos incluidos se muestran en la tabla 1.

Tabla 1. Artículos considerados en la revisión de investigaciones en enfermedades musculoesqueléticas por el trabajo.

\begin{tabular}{|c|c|c|c|}
\hline Referencia & País & $\begin{array}{c}\text { Tipo } \\
\text { de publicación }\end{array}$ & $\begin{array}{c}\text { Tipo } \\
\text { de ocupación }\end{array}$ \\
\hline Al-Rawi et al., 2018 & Emiratos Árabes Unidos & $\mathrm{a}$ & $\mathrm{x}$ \\
\hline $\begin{array}{l}\text { Alyahya, Algarzaie, Alsubeh, \& } \\
\text { Khounganian, } 2018\end{array}$ & Arabia Saudita & a & $\mathrm{x}$ \\
\hline Bao \& Lin, 2018 & Estados Unidos & $\mathrm{a}$ & $\mathrm{z}$ \\
\hline Barros-Gomes et al., 2019 & Estados Unidos & $\mathrm{a}$ & $\mathrm{x}$ \\
\hline Benjamin \& Meisinger, 2018 & Estados Unidos & $\mathrm{d}$ & $\mathrm{x}$ \\
\hline $\begin{array}{l}\text { Besharati, Daneshmandi, Zareh, } \\
\text { Fakherpour, \& Zoaktafi, } 2018\end{array}$ & Irán & a & $\mathrm{y}$ \\
\hline $\begin{array}{l}\text { Botta, Presoto, Wajngarten, } \\
\text { Campos, \& Garcia, } 2018\end{array}$ & Estados Unidos & a & $\mathrm{x}$ \\
\hline Brandt et al., 2018 & Dinamarca & $\mathrm{a}$ & $\mathrm{Z}$ \\
\hline Bulduk, 2019 & Turquía & $\mathrm{a}$ & $\mathrm{Z}$ \\
\hline $\begin{array}{l}\text { Cardenas-Trowers, Kjellsson, \& } \\
\text { Hatch, } 2018\end{array}$ & Estados Unidos & $\mathrm{b}$ & $\mathrm{w}$ \\
\hline $\begin{array}{l}\text { Catanzarite, Tan-Kim, \& } \\
\text { Menefee, } 2018\end{array}$ & Estados Unidos & d & $\mathrm{w}$ \\
\hline $\begin{array}{l}\text { Catanzarite, Tan-Kim, } \\
\text { Whitcomb, \& Menefee, } 2018\end{array}$ & Estados Unidos & $d$ & $\mathrm{w}$ \\
\hline Celik et al., 2018 & Turquía & $\mathrm{a}$ & $\mathrm{y}$ \\
\hline Chatterjee \& Sahu, 2018 & India & $\mathrm{a}$ & $\mathrm{Z}$ \\
\hline $\begin{array}{l}\text { Comper, Dennerlein, } \\
\text { Evangelista, Rodrigues da Silva, } \\
\text { \& Padula, } 2017\end{array}$ & Brasil & a & $\mathrm{z}$ \\
\hline Coskun Beyan et al., 2018 & Turquía & $\mathrm{b}$ & $\mathrm{Z}$ \\
\hline $\begin{array}{l}\text { Dabholkar, Yardi, Dabholkar, } \\
\text { Velankar, \& Ghuge, } 2017\end{array}$ & India & a & $\mathrm{w}$ \\
\hline $\begin{array}{l}\text { Dalager, Hojmark, Jensen, } \\
\text { Sogaard, \& Andersen, } 2019\end{array}$ & Dinamarca & a & $\mathrm{w}$ \\
\hline $\begin{array}{l}\text { Davila, Meltzer, Hallbeck, } \\
\text { Stone, \& Money, } 2019\end{array}$ & Estados Unidos & a & $\mathrm{w}$ \\
\hline De Sio et al., 2018 & Italia & $\mathrm{c}$ & $\mathrm{x}$ \\
\hline Depreli \& Angin, 2018 & Chipre & $\mathrm{a}$ & $\mathrm{y}$ \\
\hline $\begin{array}{l}\text { Dianat, Bazazan, Souraki Azad, } \\
\text { \& Salimi, } 2018\end{array}$ & Irán & a & $\mathrm{w}$ \\
\hline Epstein et al., 2018 & Estados Unidos & $\mathrm{c}$ & $\mathrm{w}$ \\
\hline Ergan, Baskurt, \& Baskurt, 2017 & Turquía & $\mathrm{a}$ & $\mathrm{x}$ \\
\hline $\begin{array}{l}\text { Fernández de Grado, Denni, } \\
\text { Musset, \& Offner, } 2019\end{array}$ & Francia & a & $\mathrm{x}$ \\
\hline $\begin{array}{l}\text { Fiodorenko-Dumas, } \\
\text { Kurkowska, \& Paprocka- } \\
\text { Borowicz, } 2018\end{array}$ & Polonia & a & $\mathrm{z}$ \\
\hline Giagio et al., 2019 & Italia & $\mathrm{a}$ & $\mathrm{W}$ \\
\hline Habib, Ziadee, Abi Younes, \& & Siria & $\mathrm{a}$ & $\mathrm{Z}$ \\
\hline
\end{tabular}




\begin{tabular}{|c|c|c|c|}
\hline Harastani, 2020 & & & \\
\hline Hakim \& Mohsen, 2017 & Egipto & $\mathrm{a}$ & $\mathrm{z}$ \\
\hline Hallbeck et al., 2017 & Estados Unidos & $\mathrm{a}$ & $\mathrm{w}$ \\
\hline $\begin{array}{l}\text { Hoe, Urquhart, Kelsall, Zamri, } \\
\text { \& Sim, } 2018\end{array}$ & Malasia & $\mathrm{c}$ & $\mathrm{y}$ \\
\hline Hossain et al., 2018 & Bangladés & $\mathrm{a}$ & $\mathrm{z}$ \\
\hline $\begin{array}{l}\text { Howarth, Hallbeck, Lemaine, } \\
\text { Singh, \& Noland, } 2019\end{array}$ & Estados Unidos & a & $\mathrm{w}$ \\
\hline $\begin{array}{l}\text { Howarth, Hallbeck, Mahabir, } \\
\text { et al., } 2019\end{array}$ & Estados Unidos & a & $\mathrm{w}$ \\
\hline Intranuovo et al., 2019 & Italia & $\mathrm{b}$ & $\mathrm{z}$ \\
\hline $\begin{array}{l}\text { Januario, Madeleine, Cid, } \\
\text { Samani, \& Oliveira, } 2018\end{array}$ & Brasil & $\mathrm{a}$ & $\mathrm{z}$ \\
\hline $\begin{array}{l}\text { Januario, Oliveira, Cid, } \\
\text { Madeleine, \& Samani, } 2017\end{array}$ & Brasil & a & $\mathrm{z}$ \\
\hline Jeong \& Choi, 2020 & Corea & $\mathrm{a}$ & $\mathrm{x}$ \\
\hline $\begin{array}{l}\text { Johnson, Zigman, Ibbotson, } \\
\text { Dennerlein, \& Kim, } 2018\end{array}$ & Estados Unidos & $\mathrm{a}$ & $\mathrm{z}$ \\
\hline $\begin{array}{l}\text { Kaup, Shivalli, Kulkarni, \& } \\
\text { Arunachalam, } 2020\end{array}$ & India & a & $\mathrm{w}$ \\
\hline Kee \& Haslam, 2019 & Corea & $\mathrm{a}$ & $\mathrm{z}$ \\
\hline Koni et al., 2018 & Italia & $\mathrm{a}$ & $\mathrm{x}$ \\
\hline $\begin{array}{l}\text { Laal, Mirzaei, Behdani, } \\
\text { Mohammadi, \& Khodami, } 2017\end{array}$ & Tailandia & a & $\mathrm{z}$ \\
\hline Labao, Faller, \& Bacayo, 2018 & Malasia & $\mathrm{a}$ & $\mathrm{z}$ \\
\hline Lallukka et al., 2020 & Finlandia & $\mathrm{a}$ & $\mathrm{Z}$ \\
\hline Lin, Barbir, \& Dennerlein, 2017 & Estados Unidos & $\mathrm{a}$ & $\mathrm{y}$ \\
\hline Lind, Forsman, \& Rose, 2020 & Suecia & $\mathrm{a}$ & $\mathrm{y}$ \\
\hline Liu et al., 2019 & China & $\mathrm{a}$ & $\mathrm{z}$ \\
\hline $\begin{array}{l}\text { Lobo, Gandarillas, Sánchez- } \\
\text { Gómez, \& Megia, } 2019\end{array}$ & España & a & $\mathrm{w}$ \\
\hline $\begin{array}{l}\text { Luger, Maher, Rieger, \& } \\
\text { Steinhilber, } 2019\end{array}$ & Alemania & $\mathrm{c}$ & $\mathrm{z}$ \\
\hline Makishima et al., 2018 & Japón & $\mathrm{a}$ & $\mathrm{z}$ \\
\hline $\begin{array}{l}\text { Meisha, Alsharqawi, Samarah, } \\
\text { \& Al-Ghamdi, } 2019\end{array}$ & Arabia Saudita & $\mathrm{a}$ & $\mathrm{x}$ \\
\hline $\begin{array}{l}\text { Minghelli, Ettro, Simao, \& } \\
\text { Mauricio, } 2019\end{array}$ & Portugal & $\mathrm{a}$ & $\mathrm{z}$ \\
\hline Mulimani et al., 2018 & Estados Unidos & $\mathrm{c}$ & $\mathrm{x}$ \\
\hline Noble \& Sweeney, 2018 & Estados Unidos & $\mathrm{d}$ & $\mathrm{x}$ \\
\hline $\begin{array}{l}\text { Nourollahi, Afshari, \& Dianat, } \\
2018\end{array}$ & Irán & a & $\mathrm{x}$ \\
\hline $\begin{array}{l}\text { Oakman, de Wind, van den } \\
\text { Heuvel, \& van der Beek, } 2017\end{array}$ & Australia & a & $\mathrm{z}$ \\
\hline Pandalai, Wheeler, \& Lu, 2017 & Estados Unidos & $\mathrm{a}$ & $\mathrm{z}$ \\
\hline $\begin{array}{l}\text { Penkala, El-Debal, \& Coxon, } \\
2018\end{array}$ & Australia & $\mathrm{a}$ & $\mathrm{y}$ \\
\hline Pereira et al., 2017 & Australia & $\mathrm{a}$ & $\mathrm{y}$ \\
\hline Petit et al., 2018 & Francia & $\mathrm{a}$ & $\mathrm{z}$ \\
\hline $\begin{array}{l}\text { Prasad, Appachu, Kamath, \& } \\
\text { Prasad, } 2017\end{array}$ & India & a & $\mathrm{x}$ \\
\hline $\begin{array}{l}\text { Rasim Ul Hasanat, Ali, Rasheed, } \\
\text { \& Khan, } 2017\end{array}$ & Pakistán & $\mathrm{a}$ & $\mathrm{y}$ \\
\hline Rasmussen et al., 2018 & Dinamarca & $\mathrm{a}$ & $\mathrm{z}$ \\
\hline
\end{tabular}




\begin{tabular}{|c|c|c|c|}
\hline Sanaeinasab et al., 2018 & Irán & $\mathrm{a}$ & $\mathrm{y}$ \\
\hline Schmalz et al., 2019 & Alemania & $\mathrm{a}$ & $\mathrm{z}$ \\
\hline Singh \& Singh, 2019 & India & $\mathrm{b}$ & $\mathrm{y}$ \\
\hline $\begin{array}{l}\text { So, Szeto, Lau, Dai, \& Tsang, } \\
2019\end{array}$ & China & $\mathrm{a}$ & $\mathrm{z}$ \\
\hline $\begin{array}{l}\text { Szczygiel, Zielonka, Metel, \& } \\
\text { Golec, } 2017\end{array}$ & Polonia & $\mathrm{c}$ & $\mathrm{z}$ \\
\hline Thetkathuek \& Meepradit, 2018 & Tailandia & $\mathrm{a}$ & $\mathrm{Z}$ \\
\hline $\begin{array}{l}\text { Tirloni, Reis, Borgatto, \& Moro, } \\
2019\end{array}$ & Brasil & $\mathrm{a}$ & $\mathrm{z}$ \\
\hline $\begin{array}{l}\text { Tsang, So, Lau, Dai, \& Szeto, } \\
2018\end{array}$ & China & a & $\mathrm{z}$ \\
\hline $\begin{array}{l}\text { Umer, Antwi-Afari, Li, Szeto, \& } \\
\text { Wong, } 2018\end{array}$ & China & $\mathrm{c}$ & $\mathrm{z}$ \\
\hline Vaisbuch et al., 2019 & Estados Unidos & $\mathrm{a}$ & $\mathrm{w}$ \\
\hline Wareluk \& Jakubowski, 2017 & Polonia & $\mathrm{a}$ & $\mathrm{x}$ \\
\hline $\begin{array}{l}\text { Wells, Kjellman, Harper, } \\
\text { Forsman, \& Hallbeck, } 2019\end{array}$ & Suecia & $\mathrm{a}$ & $\mathrm{w}$ \\
\hline Yan et al., 2017 & China & $\mathrm{a}$ & $\mathrm{x}$ \\
\hline Zafar \& Almosa, 2019 & Arabia Saudita & $\mathrm{a}$ & $\mathrm{x}$ \\
\hline
\end{tabular}

Se identificó que el país de mayor prevalencia fue Estados Unidos con 23,07\% (18) con investigaciones originales en su mayoría. Los países europeos representaron el 24,35\% (19) de todos los estudios, destacándose también en investigaciones originales. Los países asiáticos publicaron 24,35\% (19) artículos. Los países del Medio Oeste contribuyeron con el 19,23\% (15) de todos los artículos. Australia contribuyó con el 3,84\% (3) de todas las investigaciones; y solo un país de América Latina apareció en la búsqueda, Brasil, con el 5,12\% (4) de los trabajos. La distribución completa por país se muestra en la tabla 2. 
Tabla 2. Distribución de artículos revisados, analizados y clasificados por país, tipo de población y ocupación.

\begin{tabular}{|c|c|c|c|c|c|c|c|c|c|}
\hline \multirow{2}{*}{ País } & \multirow{2}{*}{ Total } & \multicolumn{4}{|c|}{ Tipo de publicación } & \multicolumn{4}{|c|}{ Tipo de ocupación } \\
\hline & & $\mathbf{a}$ & $\mathbf{b}$ & $\mathbf{c}$ & $\mathbf{d}$ & $\mathbf{w}$ & $\mathbf{x}$ & $\mathbf{y}$ & $\mathbf{Z}$ \\
\hline Estados Unidos & 18 & 11 & 1 & 2 & 4 & 9 & 5 & 1 & 3 \\
\hline China & 5 & 4 & - & 1 & - & - & 1 & - & 4 \\
\hline India & 5 & 4 & 1 & - & - & 2 & 1 & 1 & 1 \\
\hline Brasil & 4 & 4 & - & - & - & - & - & - & 4 \\
\hline Irán & 4 & 4 & - & - & - & 1 & 1 & 2 & - \\
\hline Italia & 4 & 2 & 1 & 1 & - & 1 & 2 & - & 1 \\
\hline Turquía & 4 & 3 & 1 & - & - & - & 1 & 1 & 2 \\
\hline Australia & 3 & 3 & - & - & - & - & - & 2 & 1 \\
\hline Dinamarca & 3 & 3 & - & - & - & 1 & - & - & 2 \\
\hline Polonia & 3 & 2 & - & 1 & - & - & 1 & - & 2 \\
\hline Arabia Saudita & 3 & 3 & - & - & - & - & 3 & - & - \\
\hline Francia & 2 & 2 & - & - & - & - & 1 & - & 1 \\
\hline Alemania & 2 & 1 & - & 1 & - & - & - & - & 2 \\
\hline Corea & 2 & 2 & - & - & - & - & 1 & - & 1 \\
\hline Malasia & 2 & 1 & - & 1 & - & - & - & 1 & 1 \\
\hline Suecia & 2 & 2 & - & - & - & 1 & - & 1 & - \\
\hline Tailandia & 2 & 2 & - & - & - & - & - & - & 2 \\
\hline Bangladés & 1 & 1 & - & - & - & - & - & - & 1 \\
\hline Chipre & 1 & 1 & - & - & - & - & - & 1 & - \\
\hline Egipto & 1 & 1 & - & - & - & - & - & - & 1 \\
\hline Finlandia & 1 & 1 & - & - & - & - & - & - & 1 \\
\hline Japón & 1 & 1 & - & - & - & - & - & - & 1 \\
\hline Pakistán & 1 & 1 & - & - & - & - & - & 1 & - \\
\hline Portugal & 1 & 1 & - & - & - & - & - & - & 1 \\
\hline Siria & 1 & 1 & - & - & - & - & - & - & 1 \\
\hline España & 1 & 1 & - & - & - & 1 & - & - & - \\
\hline Emiratos Árabes Unidos & 1 & 1 & - & - & - & - & 1 & - & - \\
\hline & 78 & 63 & 4 & 7 & 4 & 16 & 18 & 11 & 33 \\
\hline
\end{tabular}

$\mathrm{a}=$ artículo de investigación original; $\mathrm{b}=$ informe breve/de casos; $\mathrm{c}=$ revisión sistemática y metanálisis; $d=$ =revisión de narrativas/bibliográficas y otros; $\mathrm{w}=$ =médico cirujano; $\mathrm{x}=$ prestadores de servicios de salud; $\mathrm{y}=$ administrativos; $\mathrm{z}=$ manufactura, construcción $\mathrm{y}$ otros.

Entre todos los estudios incluidos en la revisión, el 52,56\% se centraron en evaluar el dolor o la incomodidad relacionados con las enfermedades musculoesqueléticas relacionadas con el trabajo (WRMD, por sus siglas en inglés) en general o en varios sitios anatómicos del cuerpo; el 21,79\% se centraron en el dolor de espalda o tronco; 14,10\% estuvieron más relacionados con dolor o malestar en el cuello; y el 11,53\% se centró en el estudio de WRMD en las extremidades superiores, incluidos los hombros (figura 1). 
Figura 1. Principal sitio anatómico estudiado.

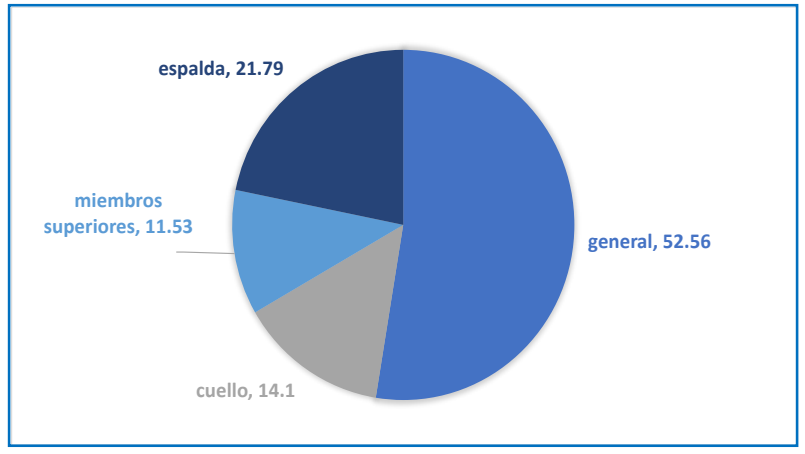

De los estudios incluidos, el 47,40\% realizó una evaluación del dolor en la población de estudio, pero solo el $37,83 \%$ de ellos, es decir el $17,94 \%$ del total, aplicó un instrumento validado para la medida del dolor relacionado con los trastornos musculoesqueléticos. De los 78 artículos encontrados, el 82,05\% no evaluó el dolor o malestar y toda la metodología estuvo enfocada a la evaluación de riesgos, sin embargo, aunque el 58,97\% de los estudios mostró una metodología clara para evaluar el factor de riesgo para la ergonomía, e incluso evaluó su intensidad, solo en uno de cada cinco estudios, el dolor fue un resultado claramente cuantificado.

Posteriormente, se aplicaron los criterios de eliminación y se seleccionaron 34 (43,58\%) estudios; esos artículos describieron claramente un método validado para medir el dolor o la intensidad de los factores de riesgo ergonómico estudiados como probablemente relacionados con el dolor en la población de estudio. Todos fueron investigaciones originales, $15(44,11 \%)$ utilizaron un instrumento validado para la evaluación del factor de riesgo, 10 $(29,41 \%)$ utilizaron un instrumento validado para la evaluación del dolor y $9(26,47 \%)$ utilizaron un método validado para ambas variables (figura 2).

Figura 2. Variable evaluada con un instrumento validado.

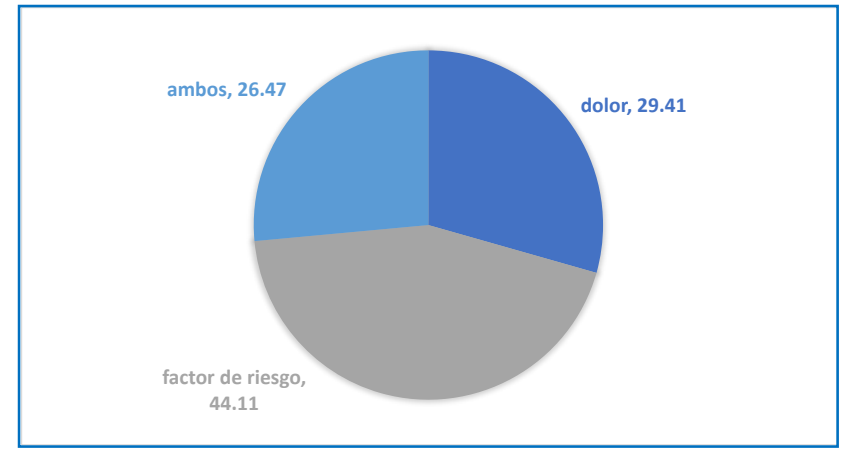

De estos artículos, solo cuatro eran ensayos clínicos. El instrumento validado más aplicado fue el Nordic Musculokeletal Questionnaire (NMQ), que se utilizó en 14 (41,17\%) de los estudios seleccionados. Sin embargo, también se aplicaron otros instrumentos validados y conocidos y de amplia aplicación en ergonomía, como RULA, OCRA, OWAS y REBA. Algunos métodos tecnológicos, como la electromiografía de superficie y la cinemática, se aplicaron en 7 (20,58\%) de los estudios. 


\section{Conclusiones}

De acuerdo con la base de datos PubMed, la participación de los países latinoamericanos en ergonomía y enfermedades musculoesqueléticas relacionadas con el trabajo es mínima. Es importante notar que en los últimos años se están haciendo muchos esfuerzos de ergonomistas, sociedades y asociaciones de países latinoamericanos que están afiliados a la Asociación Internacional de Ergonomía (IEA), sin embargo, se requiere fortalecer las investigaciones basadas en las necesidades específicas en ergonomía en esta población. Es urgente producir más investigación y conocimiento científico alineado con los estándares internacionales de metodología científica que los haga disponibles en bases de datos de mayor alcance. Por otro lado, este trabajo se basó únicamente en la base de datos PubMed, pero muchas otras bases de datos como Scopus, Web of Science o ScienceDirect, podrían tener más investigación de países latinoamericanos. Además, este trabajo se basó únicamente en las publicaciones de los últimos tres años, no obstante, los resultados de este trabajo pueden reflejar una parte de la realidad; una revisión sistemática basada en más bases de datos podría dar un punto de vista más confiable sobre esta situación.

El dolor es la principal manifestación clínica de las WRMD, este síntoma puede ser un elemento muy importante en la valoración del impacto de la ergonomía en la salud del trabajador, desde un punto de vista integral. La relevancia de las proporciones mostradas en los resultados radica en que todos los estudios incluidos en la revisión consideraron el dolor como una consecuencia inherente de los trastornos musculoesqueléticos, pero solo unos pocos lo midieron con un método validado. Podría ser comprensible que la investigación centrada en la prevención considere la presencia de dolor como un fracaso de los métodos preventivos, pero la única forma de asegurar que no hubo dolor en la población expuesta al riesgo es tomando una medida. La cuantificación se puede lograr mediante encuestas, como lo son la Escala Analógica Visual (EVA, por sus siglas en inglés) y el Cuestionario Nórdico Musculoesquelético (NMQ, por sus siglas en inglés), aunque la evaluación del dolor se puede lograr de muchas formas dependiendo de la disciplina que esté realizando el estudio. El NMQ es un cuestionario estandarizado que se utiliza para comparar las quejas generales de la espalda baja, el cuello, los hombros y el sistema musculoesquelético y fue diseñado para su uso en estudios epidemiológicos, no para establecer un diagnóstico clínico (Crawford, 2007). No obstante, en cualquier caso, la percepción del paciente-trabajador es insustituible para tener una cuantificación fiable del dolor, como pueden hacer la EVA y otros métodos validados. Por ejemplo, en los estudios que incluyen exploración física, un método como EVA podría ser necesario para hacer medible el dolor, en lugar de describir solo la presencia o ausencia del dolor; es decir, proporcionar precisión.

Otras disciplinas cuyo ámbito profesional no incluye las habilidades clínicas, de todas formas podrían medir el dolor con un método validado como dice el trabajador en una encuesta, pero siempre con una cuantificación del dolor fiable y reproducible. Cuando no se sigue esta metodología, puede resultar complicado abordar la información desde un punto de vista integral que podría ser aplicado por muchas disciplinas, principalmente las disciplinas de la salud. Por ejemplo, las revisiones sistemáticas y el metanálisis son herramientas científicas muy valiosas para sustentar las directrices y las decisiones epidemiológicas. Sin embargo, la falta de métodos validados y la valoración sólida de las variables consideradas en 
los estudios, las convierten en un riesgo de sesgo para ser incluidas en revisiones sistemáticas. Esto significa que el esfuerzo de los investigadores podría resultar aislado de la población de estudio, en lugar de ser parte de un nuevo conocimiento unificado más grande con valor clínico.

Como lo hicieron muchos de los estudios incluidos en esta revisión, la evaluación del dolor podría brindar información confiable para determinar si realmente se requieren cambios en el diseño de la estación de trabajo o en el ambiente de trabajo. Sin embargo, todos los elementos que permitan alcanzar un mayor nivel de precisión, confiabilidad y reproducibilidad, podrían ayudar a nuevas investigaciones a determinar, con un impacto aún mayor en la salud del trabajador, la relación entre ergonomía, WRMD y dolor. 


\section{Referencias}

Al-Rawi, N. H., Yousef, H., Khamis, M., Belkadi, O., Ahmed, S., \& Ali, S. (2018). Vertebral malalignment among male dentists with work-related musculoskeletal pain in the United Arab Emirates. The Journal of Contemporary Dental Practice, 19(7), 773-777. https://www.thejcdp.com/doi/pdf/10.5005/jp-journals-10024-2335

Alyahya, F., Algarzaie, K., Alsubeh, Y., \& Khounganian, R. (2018). Awareness of ergonomics \& work-related musculoskeletal disorders among dental professionals and students in Riyadh, Saudi Arabia. Journal of Physical Therapy Science, 30(6), 770-776. https://doi.org/10.1589/jpts.30.770

Bao, S., \& Lin, J. H. (2018). An investigation into four different sit-stand workstations use schedules. Ergonomics, 61(2), 243-254. https://doi.org/10.1080/00140139.2017.1353139

Barros-Gomes, S., Orme, N., Nhola, L. F., Scott, C., Helfinstine, K., Pislaru, S. V, ... Pellikka, P. A. (2019). Characteristics and consequences of work-related musculoskeletal pain among cardiac sonographers compared with peer employees: A multisite cross-sectional study. Journal of the American Society of Echocardiography: Official Publication of the American Society of Echocardiography, 32(9), 1138-1146. https://doi.org/10.1016/j.echo.2019.04.416

Benjamin, J. L., \& Meisinger, Q. C. (2018). Ergonomics in the development and prevention of musculoskeletal injury in interventional radiologists. Techniques in Vascular and Interventional Radiology, 21(1), 16-20. https://doi.org/10.1053/j.tvir.2017.12.004

Besharati, A., Daneshmandi, H., Zareh, K., Fakherpour, A., \& Zoaktafi, M. (2018). Work-related musculoskeletal problems and associated factors among office workers. International Journal of Occupational Safety and Ergonomics, 1-7. https://doi.org/10.1080/10803548.2018.1501238

Botta, A. C., Presoto, C. D., Wajngarten, D., Campos, J. A. D. B., \& Garcia, P. P. N. S. (2018). Perception of dental students on risk factors of musculoskeletal disorders. European Journal of Dental Education: Official Journal of the Association for Dental Education in Europe, 22(4), 209-214. https://doi.org/10.1111/eje.12328

Brandt, M., Madeleine, P., Samani, A., Ajslev, J. Z., Jakobsen, M. D., Sundstrup, E., \& Andersen, L. L. (2018). Effects of a participatory ergonomics intervention with wearable technical measurements of physical workload in the construction industry: Cluster randomized controlled trial. Journal of Medical Internet Research, 20(12), e10272. https://doi.org/10.2196/10272

Bulduk, E. O. (2019). Work-related stress levels and musculoskeletal disorders among municipal solid waste collectors in Ankara. Work (Reading, Mass.), 63(3), 427-433. https://doi.org/10.3233/WOR-192949

Cardenas-Trowers, O., Kjellsson, K., \& Hatch, K. (2018). Ergonomics: Making the OR a comfortable place. International Urogynecology Journal, 29(7), 1065-1066. https://doi.org/10.1007/s00192-018-3674-7

Catanzarite, T., Tan-Kim, J., \& Menefee, S. A. (2018). Ergonomics in gynecologic surgery. Current Opinion in Obstetrics \& Gynecology, 30(6), 432-440. https://doi.org/10.1097/GCO.0000000000000502

Catanzarite, T., Tan-Kim, J., Whitcomb, E. L., \& Menefee, S. (2018). Ergonomics in surgery: A review. Female Pelvic Medicine \& Reconstructive Surgery, 24(1), 1-12. https://doi.org/10.1097/SPV.0000000000000456

Celik, S., Celik, K., Dirimese, E., Tasdemir, N., Arik, T., \& Buyukkara, I. (2018). Determination of pain in musculoskeletal system reported by office workers and the pain risk factors. 
International Journal of Occupational Medicine and Environmental Health, 31(1), 91-111. https://doi.org/10.13075/ijomeh.1896.00901

Chatterjee, A., \& Sahu, S. (2018). A physiological exploration on operational stance and occupational musculoskeletal problem manifestations amongst construction labourers of West Bengal, India. Journal of Back and Musculoskeletal Rehabilitation, 31(4), 775-783. https://doi.org/10.3233/BMR-170935

Comper, M. L. C., Dennerlein, J. T., Evangelista, G. D. S., Rodrigues da Silva, P., \& Padula, R. S. (2017). Effectiveness of job rotation for preventing work-related musculoskeletal diseases: A cluster randomised controlled trial. Occupational and Environmental Medicine, 74(8), 545552. https://doi.org/10.1136/oemed-2016-104077

Coskun Beyan, A., Demiral, Y., Dilek, B., Alici, N. S., Bediz, C., \& Cimrin, A. (2018). Skydiving and the risk of cervical disc herniation. International Journal of Occupational Medicine and Environmental Health, 31(2), 243-249. https://doi.org/10.13075/ijomeh.1896.01032

Crawford, J. O. (2007). The Nordic Musculoskeletal Questionnaire. Occupational Medicine, 57(4), 300-301. https://doi.org/10.1093/occmed/kqmo36

Dabholkar, T., Yardi, S., Dabholkar, Y. G., Velankar, H. K., \& Ghuge, G. (2017). A survey of workrelated musculoskeletal disorders among otolaryngologists. Indian Journal of Otolaryngology and Head and Neck Surgery: Official Publication of the Association of Otolaryngologists of India, 69(2), 230-238. https://doi.org/10.1007/s12070-017-1106-5

Dalager, T., Hojmark, A., Jensen, P. T., Sogaard, K., \& Andersen, L. N. (2019). Using an intervention mapping approach to develop prevention and rehabilitation strategies for musculoskeletal pain among surgeons. BMC Public Health, 19(1), 320. https://doi.org/10.1186/s12889-019-6625-4

Davila, V. J., Meltzer, A. J., Hallbeck, M. S., Stone, W. M., \& Money, S. R. (2019). Physical discomfort, professional satisfaction, and burnout in vascular surgeons. Journal of Vascular Surgery, 7o(3), 913-920.e2. https://doi.org/10.1016/j.jvs.2018.11.026

De Sio, S., Traversini, V., Rinaldo, F., Colasanti, V., Buomprisco, G., Perri, R., ... Guerra, F. (2018). Ergonomic risk and preventive measures of musculoskeletal disorders in the dentistry environment: An umbrella review. PeerJ, 6, e4154. https://doi.org/10.7717/peerj.4154

Depreli, O., \& Angin, E. (2018). Review of scapular movement disorders among office workers having ergonomic risk. Journal of Back and Musculoskeletal Rehabilitation, 31(2), 371-380. https://doi.org/10.3233/BMR-170790

Dianat, I., Bazazan, A., Souraki Azad, M. A., \& Salimi, S. S. (2018). Work-related physical, psychosocial and individual factors associated with musculoskeletal symptoms among surgeons: Implications for ergonomic interventions. Applied Ergonomics, 67, 115-124. https://doi.org/10.1016/j.apergo.2017.09.011

Epstein, S., Sparer, E. H., Tran, B. N., Ruan, Q. Z., Dennerlein, J. T., Singhal, D., \& Lee, B. T. (2018). Prevalence of work-related musculoskeletal disorders among surgeons and interventionalists: A systematic review and meta-analysis. JAMA Surgery, 153(2), e174947. https://doi.org/10.1001/jamasurg.2017.4947

Ergan, M., Baskurt, F., \& Baskurt, Z. (2017). The examination of work-related musculoskeletal discomforts and risk factors in veterinarians. Arhiv Za Higijenu Rada i Toksikologiju, 68(3), 198-205. https://doi.org/10.1515/aiht-2017-68-3011

Fernández de Grado, G., Denni, J., Musset, A.-M., \& Offner, D. (2019). Back pain prevalence, intensity and associated factors in French dentists: A national study among 1004 
professionals. European Spine Journal, 28(11), 2510-2516. https://doi.org/10.1007/s00586-019-06080-4

Fiodorenko-Dumas, Z., Kurkowska, A., \& Paprocka-Borowicz, M. (2018). Spine pain in the firefighter profession. Medycyna Pracy, 69(4), 365-373. https://doi.org/10.13075/mp.5893.00679

Giagio, S., Volpe, G., Pillastrini, P., Gasparre, G., Frizziero, A., \& Squizzato, F. (2019). A preventive program for work-related musculoskeletal disorders among surgeons: Outcomes of a randomized controlled clinical trial. Annals of Surgery, 270(6), 969-975. https://doi.org/10.1097/SLA.0000000000003199

Habib, R. R., Ziadee, M., Abi Younes, E., \& Harastani, H. (2020). Syrian refugee child workers: Gender differences in ergonomic exposures and musculoskeletal health. Applied Ergonomics, 83, 102983. https://doi.org/10.1016/j.apergo.2019.102983

Hakim, S., \& Mohsen, A. (2017). Work-related and ergonomic risk factors associated with low back pain among bus drivers. The Journal of the Egyptian Public Health Association, 92(3), 195-201. https://pubmed.ncbi.nlm.nih.gov/30341998/

Hallbeck, M. S., Lowndes, B. R., Bingener, J., Abdelrahman, A. M., Yu, D., Bartley, A., \& Park, A. E. (2017). The impact of intraoperative microbreaks with exercises on surgeons: A multicenter cohort study. Applied Ergonomics, 60, 334-341. https://pubmed.ncbi.nlm.nih.gov/28166893/

Hoe, V. C., Urquhart, D. M., Kelsall, H. L., Zamri, E. N., \& Sim, M. R. (2018). Ergonomic interventions for preventing work-related musculoskeletal disorders of the upper limb and neck among office workers. The Cochrane Database of Systematic Reviews, 10, CDoo8570. https://doi.org/10.1002/14651858.CDoo8570.pub3

Hossain, M. D., Aftab, A., Al Imam, M. H., Mahmud, I., Chowdhury, I. A., Kabir, R. I., \& Sarker, M. (2018). Prevalence of work-related musculoskeletal disorders (WMSDs) and ergonomic risk assessment among readymade garment workers of Bangladesh: A cross sectional study. PloS One, 13(7), e0200122. https://doi.org/10.1371/journal.pone.0200122

Howarth, A. L., Hallbeck, M. S., Lemaine, V., Singh, D. J., \& Noland, S. S. (2019). Work-related musculoskeletal discomfort and injury in craniofacial and maxillofacial surgeons. The $\begin{array}{llll}\text { Journal of } & \text { Craniofacial } & \text { Surgery, } & \text { 1982-1985. }\end{array}$ https://doi.org/10.1097/SCS.0000000000005631

Howarth, A. L., Hallbeck, S., Mahabir, R. C., Lemaine, V., Evans, G. R. D., \& Noland, S. S. (2019). Work-related musculoskeletal discomfort and injury in microsurgeons. Journal of Reconstructive Microsurgery, 35(5), 322-328. https://doi.org/10.1055/s-0038-1675177

Intranuovo, G., De Maria, L., Facchini, F., Giustiniano, A., Caputi, A., Birtolo, F., \& Vimercati, L. (2019). Risk assessment of upper limbs repetitive movements in a fish industry. BMC Research Notes, 12(1), 354. https://doi.org/10.1186/s13104-019-4392-Z

Januario, L. B., Madeleine, P., Cid, M. M., Samani, A., \& Oliveira, A. B. (2018). Can exposure variation be promoted in the shoulder girdle muscles by modifying work pace and inserting pauses during simulated assembly work? Applied Ergonomics, 66, 151-160. https://doi.org/10.1016/j.apergo.2017.08.011

Januario, L. B., Oliveira, A. B., Cid, M. M., Madeleine, P., \& Samani, A. (2017). The coordination of shoulder girdle muscles during repetitive arm movements at either slow or fast pace among women with or without neck-shoulder pain. Human Movement Science, 55, 287-295. https://doi.org/10.1016/j.humov.2017.09.002

Jeong, Y.-J., \& Choi, J.-S. (2020). The effect of indirect vision skills on head and shoulder posture amongst Korean dental hygienists. European Journal of Dental Education: Official Journal 
of the Association for Dental Education in Europe, 24(1), 17-25. https://doi.org/10.1111/eje.12463

Johnson, P. W., Zigman, M., Ibbotson, J., Dennerlein, J. T., \& Kim, J. H. (2018). A randomized controlled trial of a truck seat intervention: Part 1-assessment of whole-body vibration exposures. Annals of Work Exposures and Health, 62(8), 990-999. https://doi.org/10.1093/annweh/wxy062

Kaup, S., Shivalli, S., Kulkarni, U., \& Arunachalam, C. (2020). Ergonomic practices and musculoskeletal disorders among ophthalmologists in India: An online appraisal. European Journal of Ophthalmology, 3o(1), 196-200. https://doi.org/10.1177/1120672118815107

Kee, D., \& Haslam, R. (2019). Prevalence of work-related musculoskeletal disorders in agriculture workers in Korea and preventative interventions. Work (Reading, Mass.), 64(4), 763-775. https://doi.org/10.3233/WOR-193038

Koni, A., Kufersin, M., Ronchese, F., Travan, M., Cadenaro, M., \& Larese Filon, F. (2018). Approach to prevention of musculoskeletal symptoms in dental students: an interventional study. La Medicina Del Lavoro, 109(4), 276-284. https://doi.org/10.23749/mdl.v109i4.6841

Laal, F., Mirzaei, R., Behdani, M. S., Mohammadi, M., \& Khodami, K. (2017). Evaluation of the influence of ergonomic intervention on the musculoskeletal disorders of Zahedan tailors. International Journal of Occupational Safety and Ergonomics, 23(3), 380-385. https://doi.org/10.1080/10803548.2016.1233674

Labao, H. C., Faller, E. M., \& Bacayo, M. F. D. (2018). "Aches and pains" of filipino migrant workers in Malaysia: A profile of work-related musculoskeletal disorders. Annals of Global Health, 84(3), 474-480. http://doi.org/10.29024/aogh.2331

Lallukka, T., Hiilamo, A., Oakman, J., Manty, M., Pietilainen, O., Rahkonen, O., ... Halonen, J. I. (2020). Recurrent pain and work disability: A record linkage study. International Archives of Occupational and Environmental Health, 93, 421-432. https://doi.org/10.1007/so0420019-01494-5

Lin, M. Y., Barbir, A., \& Dennerlein, J. T. (2017). Evaluating biomechanics of user-selected sitting and standing computer workstation. Applied Ergonomics, 65, 382-388. https://doi.org/10.1016/j.apergo.2017.04.006

Lind, C. M., Forsman, M., \& Rose, L. M. (2020). Development and evaluation of RAMP II - a practitioner's tool for assessing musculoskeletal disorder risk factors in industrial manual handling. Ergonomics, 1-28. https://doi.org/10.1080/00140139.2019.1710576

Liu, Y. M., Xiao, L. W., Zhou, H., Xie, C. J., Huang, L. L., \& Liu, Y. M. (2019). An analysis of workrelated musculoskeletal disorders and ergonomic loads in male welders in shipbuilding industry. Chinese journal of industrial hygiene and occupational diseases, 37(3), 201-206. https://pubmed.ncbi.nlm.nih.gov/31189242/

Llaneza Álvarez, F. J. (2009). Ergonomía y Psicosociología Aplicada. En F. J. Llaneza Álvarez (Ed.), Ergonomía y Psicosociología Aplicada. Manual para la formación del especialista (15a ed., pp. 27-107). Valladolid, España.: Lex Nova.

Lobo, D., Gandarillas, M. A., Sánchez-Gómez, S., \& Megia, R. (2019). Work-related musculoskeletal symptoms in otorhinolaryngology and their relationship with physical activity. A nationwide survey. The Journal of Laryngology and Otology, 133(8), 713-718. https://doi.org/10.1017/So022215119001452

Luger, T., Maher, C. G., Rieger, M. A., \& Steinhilber, B. (2019). Work-break schedules for preventing musculoskeletal symptoms and disorders in healthy workers. The Cochrane 


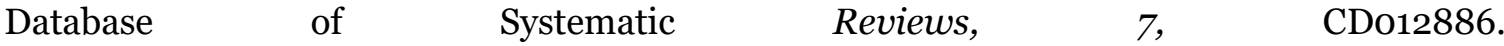
https://doi.org/10.1002/14651858.CD012886.pub2

Makishima, M., Fujino, Y., Kubo, T., Izumi, H., Uehara, M., Oyama, I., \& Matsuda, S. (2018). Validity and responsiveness of the work functioning impairment scale (WFun) in workers with pain due to musculoskeletal disorders. Journal of Occupational Health, 6o(2), 156-162. https://doi.org/10.1539/joh.17-0166-OA

Meisha, D. E., Alsharqawi, N. S., Samarah, A. A., \& Al-Ghamdi, M. Y. (2019). Prevalence of workrelated musculoskeletal disorders and ergonomic practice among dentists in Jeddah, Saudi Arabia. Clinical, Cosmetic and Investigational Dentistry, 11, 171-179. https://doi.org/10.2147/CCIDE.S204433

Minghelli, B., Ettro, N., Simao, J., \& Mauricio, K. (2019). Work-related self-reported musculoskeletal disorders in hypermarket cashiers: A study in south of Portugal. La Medicina Del Lavoro, 11O(3), 191-201. https://doi.org/10.23749/mdl.v110i3.7771

Mulimani, P., Hoe, V. C., Hayes, M. J., Idiculla, J. J., Abas, A. B., \& Karanth, L. (2018). Ergonomic interventions for preventing musculoskeletal disorders in dental care practitioners. The Cochrane Database of Systematic Reviews, 10, CDo11261. https://doi.org/10.1002/14651858.CDo11261.pub2

Noble, N. L., \& Sweeney, N. L. (2018). Barriers to the use of assistive devices in patient handling. Workplace Health \& Safety, 66(1), 41-48. https://doi.org/10.1177/2165079917697216

Nourollahi, M., Afshari, D., \& Dianat, I. (2018). Awkward trunk postures and their relationship with low back pain in hospital nurses. Work (Reading, Mass.), 59(3), 317-323. https://doi.org/10.3233/WOR-182683

Oakman, J., de Wind, A., van den Heuvel, S. G., \& van der Beek, A. J. (2017). Work characteristics predict the development of multi-site musculoskeletal pain. International Archives of Occupational and Environmental Health, 9o(7), 653-661. https://doi.org/10.1007/s00420017-1228-9

Pandalai, S. P., Wheeler, M. W., \& Lu, M.-L. (2017). Non-chemical risk assessment for lifting and low back pain based on bayesian threshold models. Safety and Health at Work, 8(2), 206211. https://doi.org/10.1016/j.shaw.2016.10.001

Penkala, S., El-Debal, H., \& Coxon, K. (2018). Work-related musculoskeletal problems related to laboratory training in university medical science students: a cross sectional survey. BMC Public Health, 18(1), 1208. https://doi.org/10.1186/s12889-018-6125-y

Pereira, M. J., Johnston, V., Straker, L. M., Sjogaard, G., Melloh, M., O’Leary, S. P., \& Comans, T. A. (2017). An investigation of self-reported health-related productivity loss in office workers and associations with individual and work-related factors using an employer's perspective. Journal of Occupational and Environmental Medicine, 59(7), e138-e144. https://doi.org/10.1097/JOM.0000000000001043

Petit, A., Bodin, J., Delarue, A., D’Escatha, A., Fouquet, N., \& Roquelaure, Y. (2018). Risk factors for episodic neck pain in workers: A 5-year prospective study of a general working population. International Archives of Occupational and Environmental Health, 91(3), 251261. https://doi.org/10.1007/s00420-017-1272-5

Prasad, D. A., Appachu, D., Kamath, V., \& Prasad, D. K. (2017). Prevalence of low back pain and carpal tunnel syndrome among dental practitioners in Dakshina Kannada and Coorg District. Indian Journal of Dental Research: Official Publication of Indian Society for Dental Research, 28(2), 126-132. https://www.ijdr.in/text.asp?2017/28/2/126/207798

Rasim Ul Hasanat, M., Ali, S. S., Rasheed, A., \& Khan, M. (2017). Frequency and associated risk factors for neck pain among software engineers in Karachi, Pakistan. JPMA. The Journal of 
the Pakistan Medical Association, 67(7), 1009-1012.

https://pubmed.ncbi.nlm.nih.gov/28770877/

Rasmussen, C. D., Hendriksen, P. R., Svendsen, M. J., Ekner, D., Hansen, K., Sorensen, O. H., \& Holtermann, A. (2018). Improving work for the body-a participatory ergonomic intervention aiming at reducing physical exertion and musculoskeletal pain among childcare workers (the TOY-project): Study protocol for a wait-list cluster-randomized controlled trial. Trials, 19(1), 411. https://doi.org/10.1186/s13063-018-2788-Z

Sanaeinasab, H., Saffari, M., Valipour, F., Alipour, H. R., Sepandi, M., Al Zaben, F., \& Koenig, H. G. (2018). The effectiveness of a model-based health education intervention to improve ergonomic posture in office computer workers: a randomized controlled trial. International Archives of Occupational and Environmental Health, 91(8), 951-962. https://doi.org/10.1007/s00420-018-1336-1

Schmalz, T., Schandlinger, J., Schuler, M., Bornmann, J., Schirrmeister, B., Kannenberg, A., \& Ernst, M. (2019). Biomechanical and metabolic effectiveness of an industrial exoskeleton for overhead work. International Journal of Environmental Research and Public Health, 16(23), 4792. https://doi.org/10.3390/ijerph16234792

Singh, H., \& Singh, L. P. (2019). Musculoskeletal disorders among insurance office employees: A case study. Work (Reading, Mass.), 64(1), 153-160. https://doi.org/10.3233/WOR-192978

So, B. C. L., Szeto, G. P. Y., Lau, R. W. L., Dai, J., \& Tsang, S. M. H. (2019). Effects of ergomotor intervention on improving occupational health in workers with work-related neck-shoulder pain. International Journal of Environmental Research and Public Health, 16(24), 5005. https://doi.org/10.3390/ijerph16245005

Szczygiel, E., Zielonka, K., Metel, S., \& Golec, J. (2017). Musculo-skeletal and pulmonary effects of sitting position - a systematic review. Annals of Agricultural and Environmental Medicine, 24(1), 8-12. https://doi.org/10.5604/12321966.1227647

Thetkathuek, A., \& Meepradit, P. (2018). Work-related musculoskeletal disorders among workers in an MDF furniture factory in eastern Thailand. International Journal of Occupational Safety and Ergonomics, 24(2), 207-217. https://doi.org/10.1080/10803548.2016.1257765

Tirloni, A. S., Reis, D. C. Dos, Borgatto, A. F., \& Moro, A. R. P. (2019). Association between perception of bodily discomfort and individual and work organizational factors in Brazilian slaughterhouse workers: a cross-sectional study. BMJ Open, 9(2), e022824. https://bmjopen.bmj.com/content/bmjopen/9/2/e022824.full.pdf

Tsang, S. M., So, B. C., Lau, R. W., Dai, J., \& Szeto, G. P. (2018). Effects of combining ergonomic interventions and motor control exercises on muscle activity and kinematics in people with work-related neck-shoulder pain. European Journal of Applied Physiology, 118(4), 751-765. https://doi.org/10.1007/s00421-018-3802-6

Umer, W., Antwi-Afari, M. F., Li, H., Szeto, G. P., \& Wong, A. Y. (2018). The prevalence of musculoskeletal symptoms in the construction industry: A systematic review and metaanalysis. International Archives of Occupational and Environmental Health, 91(2), 125144. https://doi.org/10.1007/s00420-017-1273-4

Vaisbuch, Y., Aaron, K. A., Moore, J. M., Vaughan, J., Ma, Y., Gupta, R., \& Jackler, R. K. (2019). Ergonomic hazards in otolaryngology. The Laryngoscope, 129(2), 370-376. https://doi.org/10.1002/lary.27496

Wareluk, P., \& Jakubowski, W. (2017). Evaluation of musculoskeletal symptoms among physicians performing ultrasound. Journal of Ultrasonography, 17(70), 154-159. https://doi.org/10.15557/JoU.2017.0023 
Wells, A. C., Kjellman, M., Harper, S. J. F., Forsman, M., \& Hallbeck, M. S. (2019). Operating hurts: A study of EAES surgeons. Surgical Endoscopy, 33(3), 933-940. https://doi.org/10.1007/s00464-018-6574-5

Westgaard, R. H., \& Winkel, J. (1997). Ergonomic intervention research for improved musculoskeletal health: A critical review. International Journal of Industrial Ergonomics, 2o(6), 463-500. https://doi.org/10.1016/So169-8141(96)0o076-5

Yan, P., Zhang, L., Li, F. Y., Yang, Y., Wang, Y. N., Huang, A. M., ... Yao, H. (2017). Evaluation of ergonomic load of clinical nursing procedures. Chinese journal of industrial hygiene and occupational diseases, 35(8), 581-584. https://europepmc.org/article/med/29081125

Zafar, H., \& Almosa, N. (2019). Prevalence of work-related musculoskeletal disorders among dental students of King Saud University, Riyadh, Kingdom of Saudi Arabia. The Journal of Contemporary Dental Practice, 2O(4), 449-453. https://pubmed.ncbi.nlm.nih.gov/31308275/ 